\title{
Guidewires for lower extremity artery angioplasty: a review
}

\author{
Roberto Lorenzoni ${ }^{1 *}$, MD, FESC, FACC; Roberto Ferraresi², MD; Marco Manzi ${ }^{3}$, MD; \\ Marco Roffi ${ }^{4}, \mathrm{MD}, \mathrm{FESC}, \mathrm{FACC}$
}

1. Cardiovascular Department, San Luca Hospital, Lucca, Italy; 2. Peripheral Interventional Unit, Humanitas Gavazzeni, Bergamo, Italy; 3. Interventional Radiology Unit, Policlinico Abano Terme, Padua, Italy; 4. Division of Cardiology, University Hospital, Geneva, Switzerland

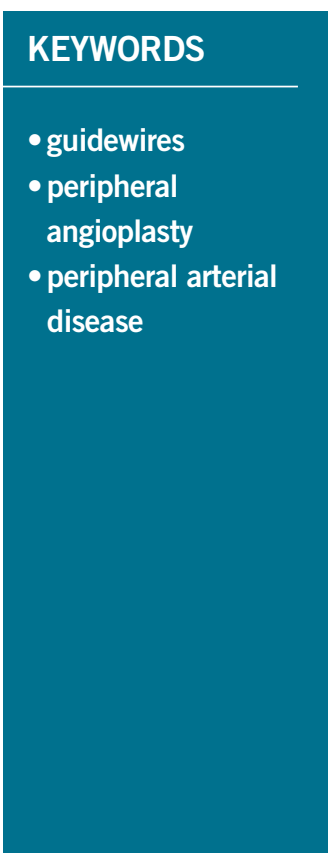

\section{Abstract}

The guidewire $(\mathrm{GW})$ is probably the most important tool for the endovascular treatment of arterial obstructions. In fact, the treatment of a lesion is only possible when the GW is passed beyond the target lesion. Lower limb percutaneous arterial revascularisation can be achieved using a variety of GWs which may differ in calibre, body, tip or coating. As the choice of an appropriate GW is critical for the success of a lower limb artery angioplasty, knowledge of the properties and performances of different GWs should be well known in order to tailor the choice of the device to the lesion characteristics and location, as well as to the intended revascularisation strategy. The aim of the present paper is to describe the constructive characteristics of GWs for lower limb arterial revascularisation, and to evaluate groups of GWs for each segment of the lower limb arterial vasculature.

*Corresponding author: UO Malattie Cardiovascolari, Ospedale San Luca, Via Lippi Francesconi, 55100 Lucca, Italy. 


\section{Introduction}

Lower limb arterial endovascular revascularisation is increasingly performed worldwide due to the rising prevalence of peripheral artery disease as well as the improvement in equipment and techniques. Guidewires (GWs) are necessary tools for any type of vascular angioplasty, allowing lesion crossing and advancement of devices such as balloons and stents beyond a lesion ${ }^{1}$. Until recently, the choice of GWs specifically designed for lower extremity artery angioplasty has been limited. For example, there were no dedicated below-the-knee (BTK) GWs and such procedures were frequently performed using coronary GWs. Currently, the spectrum of GWs designed for lower limb interventions has broadened but their characteristics have not been adequately summarised. The aim of this paper is to describe the constructive properties and performance of GWs dedicated to lower limb arterial interventions in order to help operators to choose the best device according to lesion characteristics and the segment affected.

\section{Properties of guidewires}

The functional properties of a GW depend on its calibre and on the constructive characteristics of the tip and the body. The characteristics of the tip are the main determinants for crossing the lesion, while the characteristics of the body are critical for the delivery of devices (e.g., balloons and stents) at the level of the lesion.

\section{WIRE CALIBRE}

GWs are first classified according to their calibre. The most widely used GWs in the lower limb arterial vasculature are $0.014 ", 0.018$ " or 0.035 " in diameter.

As a general rule, the larger the calibre, the greater the support, torque control and pushability; and, the smaller the calibre, the greater the flexibility and trackability of the GW. The term "crossing GW" can be applied to a GW specifically designed to traverse challenging lesions. The principal features of a crossing $\mathrm{GW}$ are a high penetration capacity and a good torque control of the tip. Often these GWs have a hydrophilic tip.

After having crossed the lesion with a crossing GW, per definition quite "aggressive", it is advisable to exchange it for a "position GW" whose principal features are a soft, non-hydrophilic tip coupled with a supportive body, to allow equipment advancement while minimising the risk of damaging the distal vasculature.

The choice of calibre of the GW must be made according to the access site and the type as well as the localisation of the lesion treated: this affects the size of all the devices used in the procedure. In fact, balloons and stents must be compatible with the GW (and the sheath) used. As a general rule, 0.035" devices are compatible with a $6 \mathrm{Fr}$ or larger sheath, 0.018 " devices can be used with a 4 or $5 \mathrm{Fr}$ sheath, and 0.014 " devices are for the most part compatible with a $4 \mathrm{Fr}$ sheath strategy. Larger calibre devices on a smaller GW (e.g., a 0.035 " compatible device advanced over a 0.018 " GW or a 0.018 " compatible device advanced over a 0.014 " GW) can be used but navigation may be more difficult.

\section{WIRE TIP}

The tip characteristics of a 0.035 " GW are limited to their general shape (i.e., straight, curved), the length of the flexible part and the coating (i.e., hydrophilic or not). The tip characteristics of 0.014 " and 0.018 " GWs include the design, the load and the coating.

GWs can have a shaping ribbon design, i.e., the core does not reach the tip of the wire, or a core-to-tip design, i.e., the core reaches the tip (Figure 1A, Figure 1B). The core-to-tip design permits a better torque control but renders the wire more aggressive (i.e., with a greater risk of vessel perforation). Usually, the diameter of a $\mathrm{GW}$ is the same throughout its length. However, some GWs have a progressive reduction of the diameter near the tip. This characteristic increases the penetration capacity. These devices are called "tapered tip" GWs (Figure 1C).

The tip load of a GW is calculated with a tester that measures the force that has to be applied to the last $10 \mathrm{~mm}$ of the tip to flex it $2 \mathrm{~mm}$ laterally. This measure is expressed in grams and gives an indication of the penetration capacity of a GW. However, as the penetration power is inversely correlated to the area of the tip of the wire, tapered GWs have a far higher penetration capacity than an equal tip load, non-tapered device.

The cover of the tip also influences the ability of the GW to cross a lesion. In particular, polymeric coating gives a hydrophilic property to the tip that becomes slippery once in contact with the bloodstream. These hydrophilic GWs are more suitable to cross tight and calcific stenosis, although they carry a higher risk of perforation. The curve of the tip of a GW has to fit the diameter of the vessel to be treated and the bends of the arterial tree in which the device has to navigate. Therefore, a proper shaping of the tip is important to enhance the performance of a $\mathrm{GW}$.

Lower limb artery diameters span from 10-12 mm of a common iliac artery to $1.5-2 \mathrm{~mm}$ of a below-the-ankle vessel. While 0.035 " hydrophilic peripheral GWs, used mainly in large vessels, are frequently pre-shaped, most 0.018 " and 0.014 " peripheral GWs have a straight-tip, shapeable design.

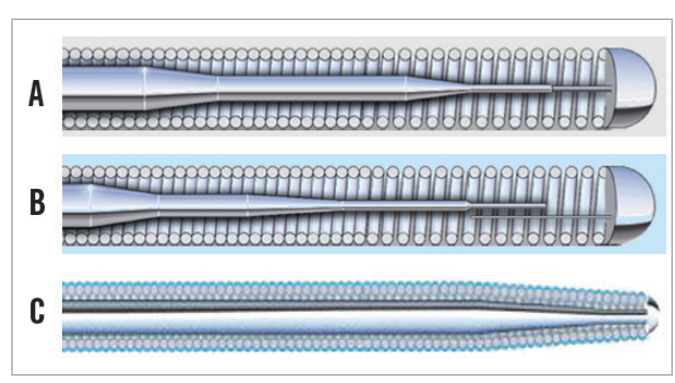

Figure 1. The two main inner designs of the tip of a guidewire. In the core-to-tip design (A) the core of the wire is directly connected to the tip: these constructive characteristics are associated with better torque control but the wire is more prone to perforate the vessel. In the shaping ribbon design (B) the core is not directly connected to the tip: in this case the wire is less aggressive but it has less torque control. Tapered tip guidewires have a progressive reduction of the diameter near the tip with a higher penetration capacity (C). 
The accuracy with which the tip of a GW can be shaped depends mainly on its inner composition. A steel core of the tip allows an accurate shaping of the tip, but subsequently the degree of the curve can only be increased. Therefore, the tip of a steel core GW has to be shaped with care and step by step, because it is nearly impossible to reverse the curves. GWs with a nitinol core are more difficult to shape because they tend to recover their original profile; however, once modified, they can be re-shaped. The tip of the GW can be shaped using the inserter or with the non-cutting edge of the blade (Figure 2).

The final bend of the tip of the GW can be a gentle, continuous curve or can comprise single or multiple acute bends (Figure 3 ). Acute bends allow a more precise tip control but may be difficult to obtain in GWs with a nitinol core. Therefore, curvilinear bends can be adequate for 0.035 " GWs with a nitinol core and appropriate for aortoiliac and femoropopliteal interventions. On the other hand, acute bends are more easily created in 0.018 " and 0.014 " GWs with a steel core and are more appropriate for BTK interventions.

\section{WIRE BODY}

A steel core gives good torque control, pushability and support, but it is more prone to be irreversibly damaged. These characteristics are ideal for a position GW. A nitinol core is associated with less torque control, pushability and support, but the wire is more kink resistant. According to the different characteristics of steel and nitinol, the core of the body of some GWs is now made of steel in the most proximal part, to have more support and torque control, and of nitinol in the more distal part, to offer flexibility and durability (e.g., Command; Abbott Vascular, Santa Clara, CA, USA). An alternative solution for a similar result is obtained in hybrid nitinol GWs: the proximal part is a super stiff shaft due to a non-hydrophilic spiral coating for better torque control and device delivery, while the most distal part is a standard hydrophilic tip for better crossing capacity (e.g., Glidewire Advantage ${ }^{\circledR}$; Terumo Corp., Tokyo, Japan).

The distance from the access site to the target lesion and the bends the GW has to pass through during its path both influence the choice of wire with respect to its body structure. Accordingly,

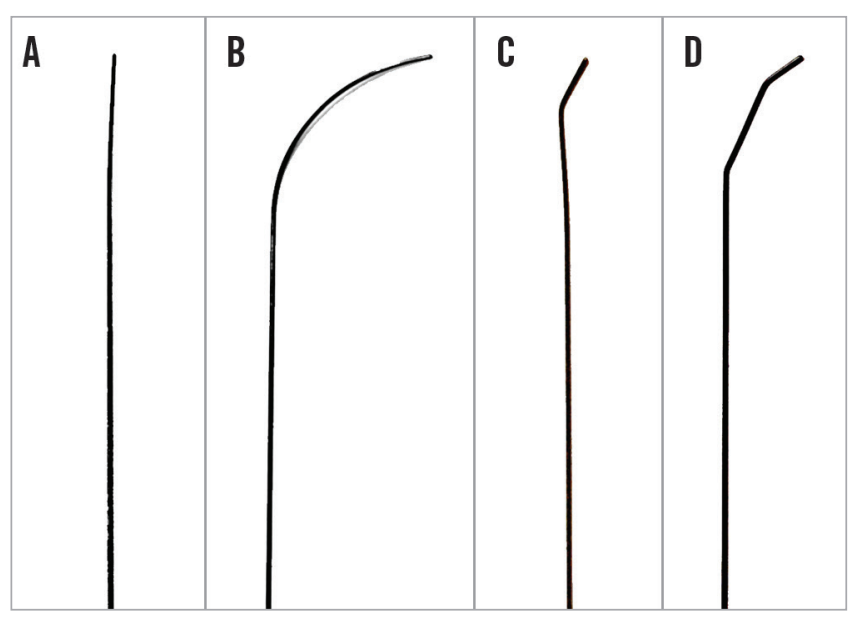

Figure 3. Different curves of the tip of the guidewire. Guidewires may be used (rarely) with a straight tip (A); however, they are best used when shaped according to the different characteristics of the vessel, i.e., with a continuous curve $(B)$ or with single $(C)$ or multiple acute bends (D).

for procedures via the arm or via a transfemoral crossover access, the support of the wire is critical and a steel core may be preferred. For an antegrade BTK angioplasty, the durability of a nitinol core may be of advantage.

In addition, the body of the GW can be covered with a hydrophilic coating that becomes slippery when wet. This constructive property confers trackability to the device.

The transition point between the body and the tip is also important. In fact, when attempting a subintimal tracking, the wire will flex at the transition point, which, therefore, should not be too far from the tip to allow a thinner loop.

\section{Principles of lesion crossing}

In lower extremity artery obstructive disease, lesions are heterogeneous in terms of vessel size, type of lesion (i.e., stenosis or occlusions), length of lesion, and degree of calcification. It is critical to

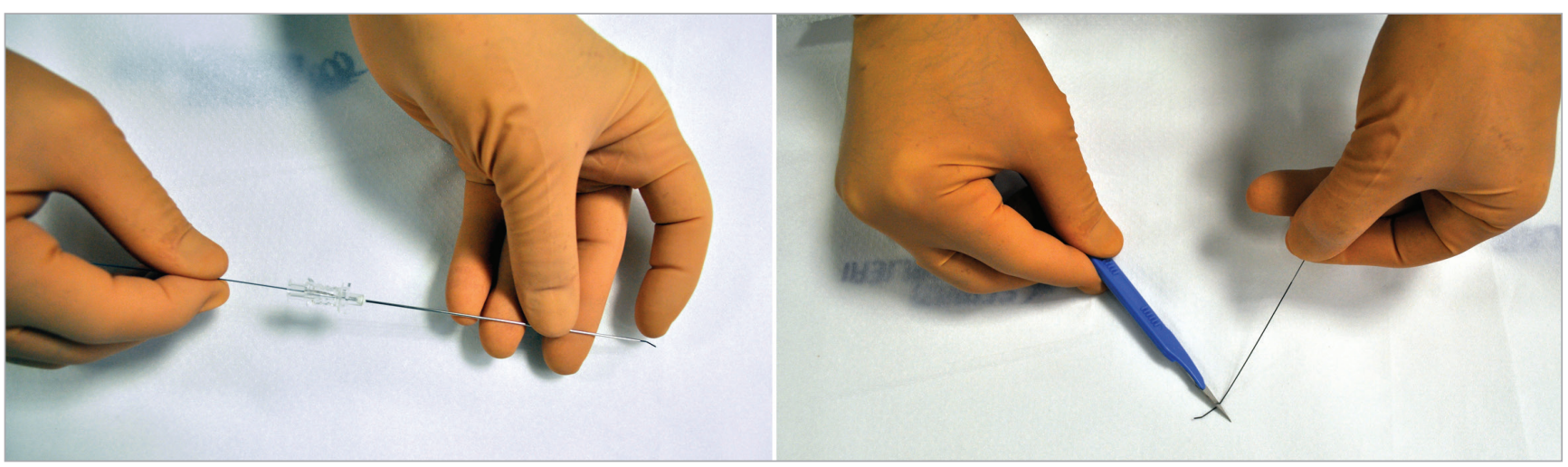

Figure 2. How to shape the tip of the guidewire. The tip of the guidewire can be shaped using the tip of the inserter (left panel) or, with caution, using the non-cutting edge of an unused blade (right panel). A curved shape can be achieved using the body of the inserter. 
differentiate GWs dedicated to lesion crossing from those allowing balloon and stent advancement once the lesion is crossed. The choice of GW calibre must be guided only by the crossing issue. Regardless of the vessel size (aortoiliac, femoropopliteal, BTK vessels) and in every vascular area, any size can be used (i.e., 0.014 ", 0.018 " or 0.035 " calibre), with the sole aim of getting into the true distal lumen (Figure 4). GWs must be tailored to the different situations that can be encountered.

In stenotic lesions, a residual true lumen with antegrade blood flow is present. Therefore, it is of paramount importance to maintain the endoluminal position during lesion crossing, avoiding subintimal tracking, especially in the presence of vessel tortuosity. In these cases, a "sliding" strategy must be pursued: a softtip GW may be rotated and slowly advanced in order to avoid producing any dissection. A flexible tip GW is preferred, while a hydrophilic coating can help in trackability (e.g., Radifocus ${ }^{\circledR}$ Guidewire M Standard [Terumo], AQUATRACK ${ }^{\mathrm{TM}}$ Regular [Cordis, Johnson \& Johnson, Warren, NJ, USA] as 0.035" GWs [Table 1]; Connect [Abbott Vascular], V-18 ${ }^{\mathrm{TM}}$ Long Taper [Boston Scientific, Marlborough, MA, USA] as 0.018" GWs [Table 2]; Command, V-14 ${ }^{\mathrm{TM}}$ Long Taper [Boston Scientific] as 0.014" GWs [Table 3]). Configuring the tip shape of smaller calibre GWs to progress within the vessel pathway is also important, and an acute, $1-2 \mathrm{~mm}$ long bend is usually adequate for 0.018 " and 0.014 " GWs.

In crossing occlusions the endoluminal approach is in any case the first choice irrespective of the length of the lesion ${ }^{2}$. The first GW to be used can be a hydrophilic 0.014 " or 0.018 " GW with a flexible tip, trying to slide into the occluded arterial lumen following the softer pathways inside the occluding material. In case of failure of this approach, a "perforating strategy" using a more aggressive GW specifically designed to traverse hostile (long and/or calcified) lesions is recommended. The principal features of these GWs are the high penetration capacity of the tip and good torque control. Such wires are often non-hydrophilic and have tapered tips with a high tip load (e.g., Connect 250T [Abbott Vascular], Astato 30 [ASAHI Intecc, Aichi, Japan], Victory ${ }^{\mathrm{TM}} 1830 \mathrm{~g}$ [Boston Scientific] as 0.018" GWs; Winn 200T [Abbott Vascular], Astato XS 20 [ASAHI Intecc], Victory ${ }^{\mathrm{TM}} 1430$
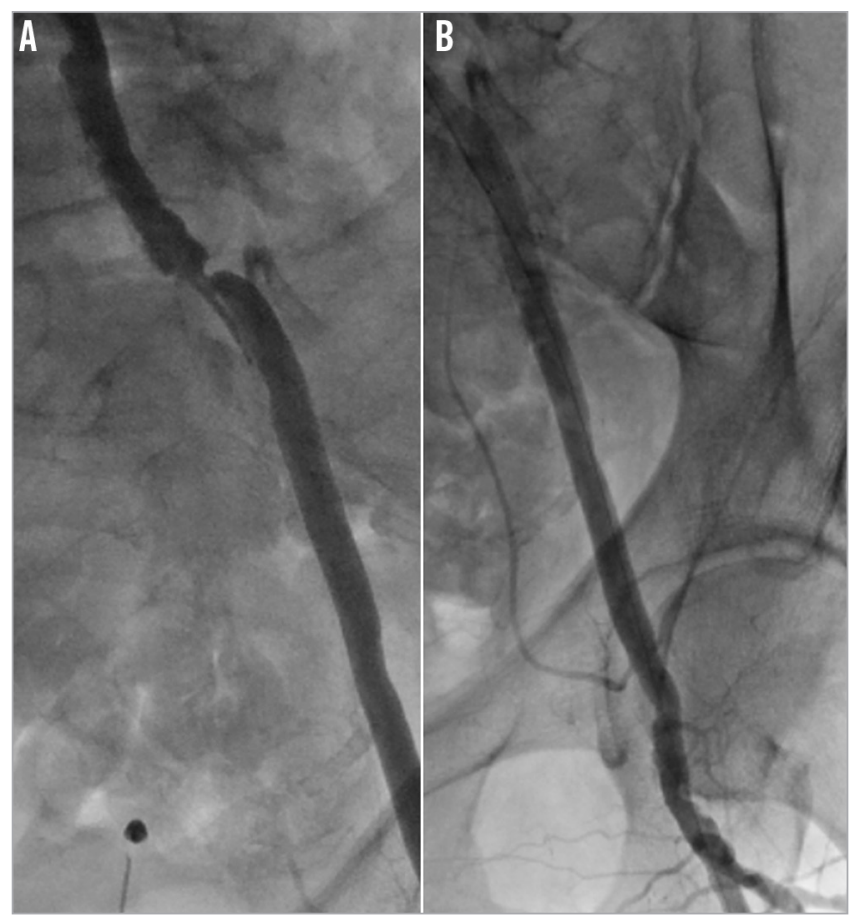

Figure 4. External iliac artery angioplasty. UL, 86-year-old male; intermittent claudication (Rutherford 3). Tight stenosis of the left external iliac artery (A). The lesion was approached by left transradial access via a $120 \mathrm{~cm}$ long sheathless catheter (SheathLessPV; ASAHI).

The stenosis was crossed with a $300 \mathrm{~cm}$ long, 0.014", hydrophilic, crossing guidewire (Command ES) advanced within a $180 \mathrm{~cm}$ long, 0.018" compatible balloon (Pacific Plus; Medtronic). After having crossed the stenosis with the balloon, the previous guidewire was exchanged for a 0.018", soft-tip, non-hydrophilic, $400 \mathrm{~cm}$ long position guidewire (Plywire). Final result after predilatation, stenting and post-dilatation as appropriate (B).

g [Boston Scientific] as 0.014" GWs [Table 1-Table 3]). Notably, the ability of a wire to cross the lesion is enhanced by advancing a balloon or a support catheter over the wire near its tip.

As the endoluminal approach has a high rate of failure in long occlusions, the subintimal approach represents an alternative option for the endovascular treatment of these lesions (Figure 5) ${ }^{3}$.

Table 1. 0.035" guidewires for lower extremity artery angioplasty.

\begin{tabular}{|l|l|c|c|c|c|c|}
\hline Producer & Model & Body core & Support & Hydrophilic tip & Flexing point & Main use \\
\hline Abbott Vascular & Supra Core & Steel & High & No & - & Position \\
\hline Boston Scientific & Amplatz Super Stiff & Steel & High & No & - & Position \\
\hline Cordis & AQUATRACK Regular & Nitinol & Low & Yes & $3 \mathrm{~cm}$ & Crossing \\
\hline Cordis & AQUATRACK Stiff & Nitinol & Medium & Yes & $3 \mathrm{~cm}$ & Crossing \\
\hline Covidien & Stiff Shaft & Nitinol & High & No & - & Position \\
\hline Terumo & Radifocus M Standard & Nitinol & Low & Yes & $3 \mathrm{~cm}$ & Crossing \\
\hline Terumo & Radifocus M Stiff & Nitinol & Medium & Yes & $3 \mathrm{~cm}$ & Crossing \\
\hline Terumo & Radifocus M Half-stiff & Nitinol & Medium & Yes & $1.5 \mathrm{~cm}$ & Crossing \\
\hline Terumo & Glidewire Advantage & Nitinol & High & Yes & $5 \mathrm{~cm}$ & Crossing \\
\hline
\end{tabular}

The table does not intend to report a complete list of guidewires available on the market but reflects the personal preferences of the authors. The flexing point pertains only to crossing guidewires suitable for the loop technique. Guidewires are listed in alphabetical order according to the producer. 
Table 2. 0.018" guidewires for lower extremity artery angioplasty.

\begin{tabular}{|l|l|c|c|c|c|c|c|c|c|}
\multicolumn{1}{|c|}{ Producer } & \multicolumn{1}{|c|}{ Model } & Body core & Support & Tip design & Tip weight & Hydrophilic tip & Tapered tip & Flexing point & Main use \\
\hline Abbott Vascular & Connect & Steel & Medium & Core to tip & $4.0 \mathrm{~g}$ & Yes & No & - & Crossing \\
\hline Abbott Vascular & Connect Flex & Steel & Medium & Core to tip & $12.0 \mathrm{~g}$ & Yes & No & $3 \mathrm{~cm}$ & Crossing \\
\hline Abbott Vascular & Connect 250T & Steel & Medium & Core to tip & $30.0 \mathrm{~g}$ & No & Yes & - & Crossing \\
\hline Abbott Vascular & Steelcore & Steel & High & Shaping ribbon & - & No & No & - & Position \\
\hline ASAHI & Treasure 12 & Steel & Medium & Core to tip & $12.0 \mathrm{~g}$ & No & No & - & Crossing \\
\hline ASAHI & Astato 30 & Steel & Medium & Core to tip & $30.0 \mathrm{~g}$ & No & Yes & - & Crossing \\
\hline Boston Scientific & Platinum Plus & Steel & High & Core to tip & $5 \mathrm{~g}$ & No & No & - & Position \\
\hline Boston Scientific & V-18 Long Taper & Steel & Medium & Core to tip & $6 \mathrm{~g}$ & Yes & No & - & Crossing \\
\hline Boston Scientific & V-18 Short Taper & Steel & Medium & Core to tip & $8 \mathrm{~g}$ & Yes & No & $8 \mathrm{~cm}$ & Crossing \\
\hline Boston Scientific & Victory 18 & Steel & Medium & Core to tip & $12-18-25-30 \mathrm{~g}$ & Yes & No & - & Crossing \\
\hline Optimed & Plywire & Steel & Medium & Core to tip & - & No & No & - & Position \\
\hline Terumo & Glidewire Advantage & Nitinol & High & Core to tip & - & Yes & No & $1 \mathrm{~cm}$ & Crossing \\
\hline
\end{tabular}

The table does not intend to report a complete list of guidewires available on the market but reflects the personal preferences of the authors. The flexing point pertains only to crossing guidewires suitable for the loop technique. Guidewires are listed in alphabetical order according to the producer.

Table 3. 0.014" guidewires for lower extremity artery angioplasty.

\begin{tabular}{|l|l|c|c|c|c|c|c|c|}
\multicolumn{1}{|c|}{ Producer } & \multicolumn{1}{|c|}{ Model } & Body core & Support & Tip design & Tip weight & Hydrophilic tip & Tapered tip & Main use \\
\hline Abbott Vascular & Command & Steel nitinol & Low & Core to tip & $2.8 \mathrm{~g}$ & Yes & No & Crossing \\
\hline Abbott Vascular & Command ES & Steel nitinol & Medium & Core to tip & $3.5 \mathrm{~g}$ & Yes & No & Crossing \\
\hline Abbott Vascular & Spartacore & Steel & High & Core to tip & $0.9 \mathrm{~g}$ & No & No & Position \\
\hline Abbott Vascular & Winn 40, 80 & Steel & Medium & Core to tip & $4.8-9.7 \mathrm{~g}$ & No & No & Crossing \\
\hline Abbott Vascular & Winn 200T & Steel & Medium & Core to tip & $13 \mathrm{~g}$ & No & Yes & Crossing \\
\hline ASAHI & Regalia XS 1.0 & Steel & Low & Core to tip & $1.0 \mathrm{~g}$ & Yes & No & Crossing \\
\hline ASAHI & Astato XS 20 & Steel & Medium & Core to tip & $20.0 \mathrm{~g}$ & No & Yes & Crossing \\
\hline ASAHI & Grand Slam & Steel & High & Core to tip & $0.7 \mathrm{~g}$ & No & No & Position \\
\hline Boston Scientific & V-14 Long Taper & High & Core to tip & $3 \mathrm{~g}$ & Yes & No & Crossing \\
\hline Boston Scientific & Victory 14 & Steel & Medium & Core to tip & $12,18,25,30 \mathrm{~g}$ & Yes & No & Crossing \\
\hline Boston Scientific & Platinum Plus & Steel & High & Core to tip & $7.1 \mathrm{~g}$ & No & No & Position \\
\hline Cook Medical & Approach CT0 6, 12, 18,25 & Steel & Medium & Core to tip & $6,12,18,25 \mathrm{~g}$ & No & No & Crossing \\
\hline Terumo & Glidewire Advantage & Nitinol & High & Core to tip & - & Yes & No & Crossing \\
\hline
\end{tabular}

The table does not intend to report a complete list of guidewires available on the market but reflects the personal preferences of the authors. Guidewires are listed in alphabetical order according to the producer.

The characteristics of an ideal GW for a subintimal crossing are a hydrophilic tip and high body support, with the flexing point located close to the tip. In fact, the short flexing tract permits the creation of a small loop and the support of the wire assures its pushability, while the hydrophilic distal tract acts as a cutting blade. The 0.035 " GW most used for subintimal recanalisation is the Radifocus ${ }^{\circledR}$ M Stiff (Terumo), with the flex point at $3 \mathrm{~cm}$ from the tip (Table 1). A GW with similar characteristics is the AQUATRACK ${ }^{\mathrm{TM}}$ Stiff (Cordis) (Table 1). When attempting a subintimal approach in BTK vessels, it is better to switch to a 0.018 " or 0.014 " GW. The 0.018 " GWs most used for subintimal tracking are the $\mathrm{V}-18^{\mathrm{TM}}$ (Boston Scientific), short taper version, where the flex point is $8 \mathrm{~cm}$ from the tip, and the Connect Flex (Abbott Vascular) with the flexing point $3 \mathrm{~cm}$ from the tip (Table 2). In case of subintimal tracking using a 0.014" GW, the Command ES (Abbott Vascular) can be used (Table 3).
In subintimal recanalisation the most challenging moment is the re-entry into the true lumen. Although specifically designed (and expensive) re-entry devices do exist, the first attempt to re-enter into the true lumen is usually made with the same GW used to progress within the subintimal space. However, when the GW used to dissect fails to find its downstream way, a second attempt with a different GW can be made. The technical characteristics of a "re-entry" GW can be summarised as follows: first, the smaller the better. In fact, it is often advisable to downsize the re-entry GW with respect to the calibre of the dissecting GW. For example, it is worth trying to re-enter the distal SFA with a 0.018 " GW after subintimal tracking and a failed re-entry with a $0.035 " \mathrm{GW}$, or to re-enter a distal tibial artery with a 0.014 " GW after a failed attempt with a 0.018 " GW. Second, a non-hydrophilic GW can be superior to a hydrophilic one, as it is less prone to progress into the dissection. Third, a GW with good torque control is needed because the tip has to be directed 


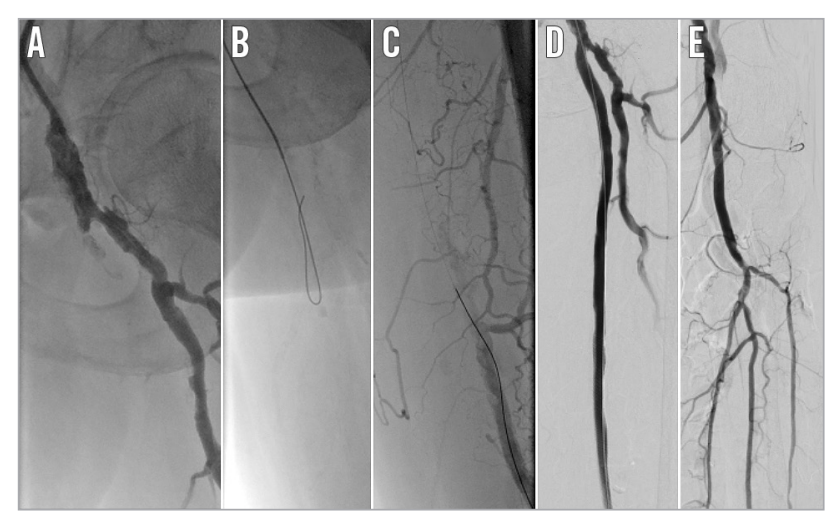

Figure 5. Superficial femoral artery angioplasty. GB, 73-year-old male; intermittent claudication (Rutherford 3). Stump occlusion of the left superficial femoral artery (A). Recanalisation was achieved with the subintimal tracking of a 0.035" hydrophilic guidewire (AQUATRACK Stiff), supported by a 4 Fr Berenstein catheter (AngioDynamics, Latham, NY, USA), using the loop technique (B). However, re-entry into the true lumen was possible only with a 0.018", tapered tip, non-hydrophilic, crossing guidewire (Astato 30) (C). After having checked the distal intraluminal position, the aggressive Astato guidewire was exchanged for a 0.018", soft-tip, non-hydrophilic position guidewire (Steelcore). Final result after predilatation, stenting and post-dilatation as appropriate $(D \& E)$.

precisely towards the true lumen or within a small hole communicating with the true lumen. Lastly, a GW with a high-grade tip load (tapering is also helpful) is necessary to perforate the intima and gain access to the true lumen (Figure 5 ).

The above-mentioned characteristics can be found in the Astato 30 or the Connect 250T (Table 2) when using 0.018" GWs, or the Astato XS 20 (Table 3) when the choice is a 0.014" GW. Before being used, the tip of a re-entry GW should be shaped adequately. A 45 degree angulation of the tip should be obtained (Figure 3 ). The length of the angulated portion should fit the vessel to be reentered, i.e., the larger the target vessel, the longer the angulated tract. Therefore, the angulated portion should be 3-4 $\mathrm{mm}$ long when trying to re-enter within an iliac artery, 2-3 $\mathrm{mm}$ long when re-entering a distal SFA or 1-2 mm long when the vessel to be reentered is a tibial artery; a secondary bending can also be added to lean the GW against the outward wall of the vessel and increase its back support (Figure 3). When in the subintimal space with the re-entry GW, a proper and accurate view should be sought with different angulations of the flat panel (also with the help of calcifications if present) in order to point the tip of the GW exactly towards the true lumen. As soon as in the true lumen, after having verified the position by angiography with a balloon catheter, these very aggressive GWs should be replaced by a position GW.

\section{Principles of lesion treatment}

After having crossed the lesion, one should reconsider whether the GW used for lesion crossing is adequate for the next steps of the procedure, namely, balloon angioplasty and possibly stenting. If not appropriate, the crossing GW should be exchanged for a position GW, according to vessel size and anatomy. When choosing a position GW it is important to take into account its calibre, tip load and the body support.

In fact, it is possible to cross an iliac lesion with a 0.014 " GW and, after predilatation, exchange it for a 0.035 " position GW for final treatment. On the other hand, it is possible to perform a subintimal dissection of a tibial artery with a $0.035 " \mathrm{GW}$ and exchange it for a 0.014 " position GW for subsequent dilatations. Similarly, dedicated GWs for crossing hostile occlusions have a very stiff tip that can damage the distal vessels. The final treatment can be carried out using a less aggressive "position GW" whose principal feature is a soft, non-hydrophilic tip. Finally, if a flexible GW fails to give adequate support for the advancement of balloons and stents at the lesion level, a "position GW", with a high-grade body support, will facilitate equipment advancement.

Therefore, the best characteristics of a GW for the treatment of a lesion are a soft non-hydrophilic tip and a very supportive body. When possible, and taking into account the size of balloons and stents $(4,5,6 \mathrm{Fr}$ compatible) in use, it is also useful to upgrade the calibre of the GW.

\section{Guidewires for aortoiliac angioplasty}

Suprainguinal angioplasty can be carried out retrogradely via ipsilateral femoral access or anterogradely via the contralateral femoral artery (crossover) or from the upper extremities, via brachial or radial arteries ${ }^{4}$. In this territory, an intraluminal approach should be pursued as much as possible because subintimal tracking carries the risk of life-threatening perforations.

When facing a stenosis in this tract, especially when working aligned with the vessel, i.e., retrogradely by ipsilateral femoral access or from the arm, either a 0.014 ", a 0.018 " or a 0.035 " $\mathrm{GW}$ may be used. In fact, the aim is to navigate within the lumen beyond the stenosis. This can be accomplished using a hydrophilic GW with a high body support. Examples among 0.035" GWs include the AQUATRACK Stiff (Table 1), a hydrophilic GW with a nitinol core, and the Radifocus M Stiff, straight or curved-tip (Terumo), which has similar features (Table 1). Alternative 0.018" GWs include the V-18 Long Taper and the Connect Flex, while amongst 0.014" GWs the Command ES may be used.

When dealing with an iliac occlusion, it may be wise first to attempt to cross intraluminally to reduce the risk of perforation. First attempts can be carried out with a hydrophilic flexible-tipped GW (e.g., Radifocus M Standard [Terumo] or AQUATRACK Regular as 0.035" GWs, Table 1; Connect, V-18 Long Taper as 0.018" GWs, Table 2; Command, V-14 Long Taper as 0.014" GWs, Table 3). In case of failure, non-hydrophilic, tapered 0.014 " GWs (e.g., Astato XS 20, Table 3) or 0.018" GWs (e.g., Astato 30 or Connect 250T, Table 2) are all valuable choices because they have good torque control and are less prone to progress subintimally. Hydrophilic 0.018" (e.g., V-18 Short Taper or Connect Flex, Table 2) or 0.035" (e.g., AQUATRACK Stiff or Radifocus M Stiff, Table 1) GWs may also be used; however, these slippery devices definitely tend to dissect and progress subintimally. 
When intraluminal tracking appears impossible, a subintimal crossing may be attempted with the above-mentioned 0.018 " or 0.035 " hydrophilic GWs. For the subintimal tracking of an iliac occlusion, an approach from above (either in crossover from the contralateral femoral artery or from the arm) is safer than a retrograde ipsilateral one because of a lower risk of dissecting the aortic wall.

Once the iliac stenosis or occlusion is crossed, the GWs may be replaced by a position GW. This is extremely important when entering from a brachial or a radial artery or when working in crossover. In fact, strong support of the GW is mandatory due to the long distance from the entry site to the target point when working from the arm, or because the devices need to cross a steep angle, when working in crossover. For this purpose, the 0.035" Supra Core ${ }^{\circledR}$ (Abbott Vascular), the Stiff Shaft (Covidien, Plymouth, MN, USA) and the Amplatz Super Stiff ${ }^{B}$ (Boston Scientific) GWs are all adequate options (Table 1). Position 0.018" GWs include the Steelcore (Abbott Vascular), the Platinum Plus ${ }^{\circledR}$ (Boston Scientific) and the Plywire (Optimed, Ettlingen, Germany), the latter being particularly useful when working from the arm because it is $400 \mathrm{~cm}$ long (Table 2). The stiff body of these GWs permits an effective tracking of the devices, while the floppy, non-hydrophilic distal part guarantees safe positioning beyond the lesion. Upsizing the calibre of the crossing GW is possible after predilatation, using a larger calibre balloon as an exchange catheter.

Examples of guidewires used in the aortoiliac segment are shown in Figure 4.

\section{Guidewires for femoropopliteal angioplasty}

In this vascular segment, when facing a stenosis, even if subocclusive, a 0.018 " GW may be the first choice, and the V-18 Short Taper (Table 2) may be a valuable option, as it possesses a hydrophilic tip with an $8 \mathrm{~g}$ tip load. Moreover, its high-grade support permits the progression of balloons and stents (and also of 0.035 " compatible devices). A good alternative with similar characteristics is the Connect Flex (Table 2).

Intraluminal recanalisation should also be attempted in SFA occlusions to minimise the risk of perforation. An attempt with the above-mentioned 0.018 " hydrophilic GWs may be tried first, while non-hydrophilic, tapered GWs such as the Astato 30 or the Connect 250T (Table 2) could be a more effective choice in case of initial failure. As mentioned, a balloon or a support catheter positioned near the tip of the GW enhances the penetration ability of the device. However, especially in the long, calcific occlusion of the SFA, it is frequently impossible to progress within the true lumen. For this reason, Amman Bolia ${ }^{3}$ in 1990 found that a subintimal new route to the distal SFA might represent an effective alternative. For subintimal angioplasty in the femoropopliteal tract, both 0.035 " and 0.018 " GWs may be used. Probably the 0.035 " GW most used for subintimal angioplasty of the SFA is the Radifocus M Stiff, while the AQUATRACK Stiff has similar characteristics (Table 1). Among the choices of 0.018 " GWs for subintimal SFA angioplasty is the V-18 Short Taper version or the Connect Flex (Table 2).

When the distal lumen is gained, position is best maintained with a position 0.035 " or 0.018 " GW, chosen from among the ones suggested for aortoiliac interventions.

When all antegrade attempts fail, a retrograde access from the SFA, distal to the re-entry point, may be attempted. A popliteal and, more recently, a distal femoral puncture ${ }^{5}$ have been suggested for this purpose. In this case, the GW has to navigate retrogradely, re-enter proximally upstream from the occlusion, and be captured by a snare or within a catheter into the sheath, to complete the angioplasty anterogradely. For that purpose either the V-18 Short Taper or the Connect Flex may be used (Table 2).

Examples of guidewires used in the femoropopliteal segment are shown in Figure 5.

\section{Guidewires for below-the-knee angioplasty}

The GWs most used for BTK angioplasty are 0.014 " ones, although some operators prefer 0.018 " GWs, and, in selected cases, even 0.035 " GWs may be used ${ }^{2}$. Nonetheless, coronary GWs have been frequently utilised in the past and are still utilised nowadays for BTK angioplasty (Table 4).

The choice of GW for BTK angioplasty mainly depends upon the characteristics of the lesion (i.e., stenosis versus occlusions, presence versus absence of calcifications) and the strategy the operator decides to pursue (i.e., intraluminal versus subintimal, antegrade versus retrograde recanalisation).

In addition, in BTK interventions, an intraluminal GW advancement should be pursued as much as possible. For this purpose, a 0.014" hydrophilic GW may be the first choice. These characteristics can be found in the Command ES or in the Victory family of GWs (Boston Scientific) (Table 3). These GWs, using a "sliding"

Table 4. Some $\mathbf{0 . 0 1 4 " ~ c o r o n a r y ~ g u i d e w i r e s ~ w h i c h ~ c a n ~ a l s o ~ b e ~ u s e d ~ f o r ~ l o w e r ~ e x t r e m i t y ~ a r t e r y ~ a n g i o p l a s t y . ~}$

\begin{tabular}{|l|l|c|c|c|c|c|c|c|}
\multicolumn{1}{|c|}{ Producer } & \multicolumn{1}{|c|}{ Model } & Body core & Support & Tip design & Tip weight & Hydrophilic tip & Tapered tip & Main use \\
\hline Abbott Vascular & Pilot 50, 150, 200 & Steel & Medium & Core to tip & $1.5,2.7,4.1 \mathrm{~g}$ & Yes & No & Crossing \\
\hline Abbott Vascular & BHW & Steel & High & Shaping ribbon & - & No & No & Position \\
\hline ASAHI & Miracle 3, 4.5, 6, 12 & Steel & Medium & Core to tip & $3,4.5,6,12 \mathrm{~g}$ & No & No & Crossing \\
\hline ASAHI & Confianza 9 & Steel & Medium & Core to tip & $9 \mathrm{~g}$ & No & Yes & Crossing \\
\hline ASAHI & Confianza Pro 9, 12, 20 & Steel & Medium & Core to tip & $9,12,20 \mathrm{~g}$ & Yes & Yes & Crossing \\
\hline Boston Scientific & PT2 MS & Nitinol & Medium & Core to tip & $2.9 \mathrm{~g}$ & Yes & No & Crossing \\
\hline
\end{tabular}

The table does not intend to report a complete list of guidewires available on the market but reflects the personal preferences of the authors. Guidewires are listed in alphabetical order according to the producer. 
strategy with delicate rotations and pushing, may succeed in finding a "soft inner pathway within the occluded arterial lumen, surrounded by stiffer walls" ${ }^{2}$. In case of failure, if needing to pursue a "perforating" approach, a stiffer, non-hydrophilic, tapered 0.014" GW like the Astato XS 20 or the Winn 200T (Table 3) can be used to take advantage of their penetration ability. Intraluminal progression can also be pursued by upgrading to 0.018 " hydrophilic GWs (e.g., V-18 Short Taper or Connect Flex, Table 2) and subsequently, according to a step-by-step approach, to 0.018 " non-hydrophilic, tapered GWs (e.g., Astato 30 or Connect 250T, Table 2).

Using these aggressive GWs the risk of perforating the vessel increases. Therefore, once the lesion is crossed, they should be replaced by a position GW using a support catheter or a balloon dilatation catheter. The characteristics of a position GW are similar to those mentioned previously, i.e., it should have a soft, nonhydrophilic tip and a high body support. Valuable 0.014 " position GWs may be the Spartacore (Abbott Vascular) or the Platinum Plus 0.014"; alternative 0.018" position GWs include the Steelcore and the Platinum Plus 0.018 ", long taper version.

If the antegrade intraluminal approach fails, a subintimal wire tracking can be attempted, with great attention to not damaging a landing zone for a distal bypass. For subintimal tracking, the previously cited Command ES or one of the Victory 0.014" hydrophilic GWs may be used, although 0.018 " GWs with a hydrophilic tip coating and a stiff body such as the V-18 Short Taper or the Connect Flex may be even more effective. Also, 0.035" hydrophilic GWs can be used for subintimal BTK angioplasty. For this purpose, the Radifocus M Half-stiff (Terumo) may be a good compromise between the effectiveness and the risk of perforation (Table 1, Table 2, Table 3). In BTK subintimal interventions, it is particularly important to maintain the loop as short and thin as possible because these vessels are small in diameter and re-entry is more difficult with larger loops.
When the antegrade progression of the GW cannot be achieved and a retrograde passage to the proximal true lumen has to be found, the pedal-plantar loop technique ${ }^{6}$, the transcollateral approach $^{7}$ and the retrograde puncture are all potentially effective strategies ${ }^{8,9}$.

The pedal-plantar loop technique is based on the possibility of navigating from dorsal to plantar circulation and vice versa through the connections of the plantar arch of the foot. Therefore, when one tibial artery is occluded and the antegrade recanalisation has failed, it is possible to try a retrograde recanalisation through the other patent tibial artery and the plantar arch. As this technique means navigating within thin and tortuous vessels, and because damaging the distal circulation can be limb-threatening, the GWs to be used in this technique should have good torque control and a hydrophilic but soft tip. Moreover, as balloons have to be advanced through the plantar arch to the distal tract of the opposite tibial artery, good support of the GW is also needed. GWs with these characteristics include the 0.014" Command and the V-14 Long Taper (Table 3).

When trying a transcollateral tracking via the perforating branches of the peroneal artery to the distal tract of the anterior or posterior tibial arteries, a soft-tip GW with less support is needed. The 0.014" Regalia XS 1.0 (ASAHI) and the Command may both be adequate (Table 3 ).

When performing a retrograde recanalisation of the tibial arteries, either with the puncture of the dorsalis pedis or the distal posterior tibial artery, a GW with good support is necessary. In fact, it is mandatory to try the rendezvous in the proximal part of the occluded vessel with the GW alone or, at most, with a balloon or a support catheter. The GW frequently chosen for these attempts is the 0.018" V-18 Short Taper (Table 2) ${ }^{2}$.

Examples of guidewires used in the BTK segment are shown in Figure 6.

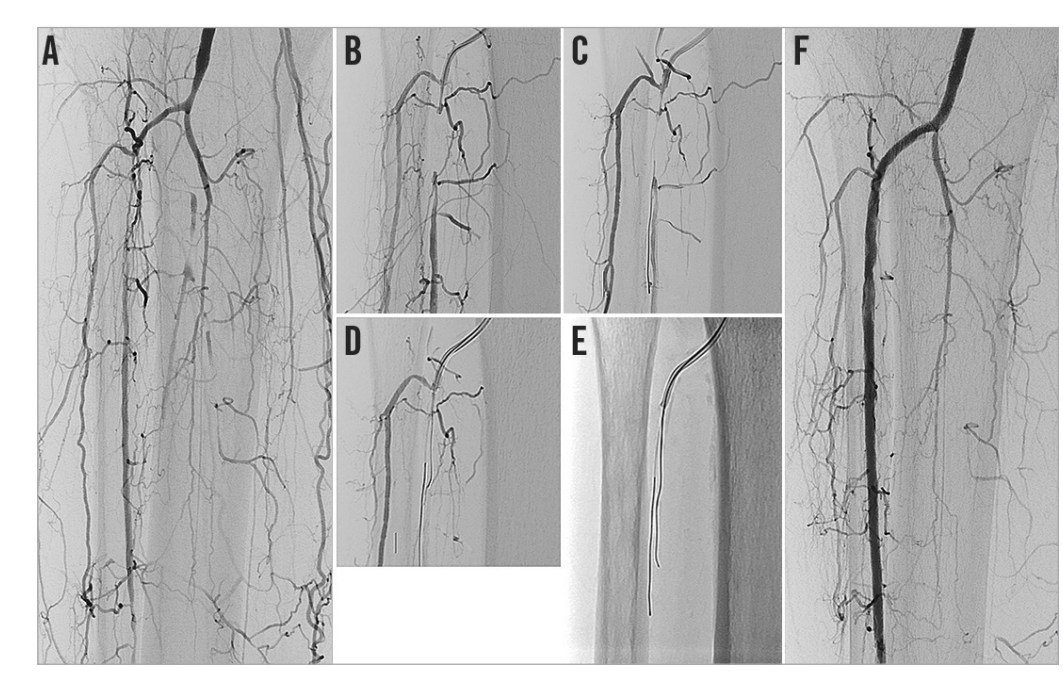

Figure 6. Tibial artery angioplasty. MB, 75-year-old male; critical limb ischaemia (Rutherford 5). Short occlusion of the proximal right anterior tibial artery (A). A 4 Fr Berenstein catheter is advanced close to the occlusion (B). A 0.014" hydrophilic guidewire with a nitinol core (Command), facing the fibrous cap, progressed into the subintimal space (C). A 0.014" hydrophilic, coronary guidewire with a steel core (Pilot 200; Abbott Vascular) was then able to navigate through the occlusion and re-enter the true lumen distal to the occlusion (D \& E). Final result after ballooning $(F)$. 


\section{Conclusions}

The choice of an appropriate GW is critical for the success of a lower limb arterial revascularisation. The properties and performance of each GW should be well known to the interventionalist in order to tailor the choice of GW to the lesion characteristics and location as well as to the revascularisation strategy.

\section{Impact on daily practice}

The guidewire (GW) is perhaps the most important tool in vascular angioplasty procedures. Knowledge of the construction and properties of GWs used in lower limb arterial revascularisation can help in choosing the right device for each lesion and each arterial segment. The calibre, tip and body characteristics, variously combined, affect the properties and contribute to the definition of crossing and position GWs. Crossing GWs are characterised by a high-grade tip load, they are often hydrophilic, and are sometimes tapered; they are used to cross the lesion. Position GWs are characterised by a soft, non-hydrophilic tip but they have a high-grade body support and are used to advance balloons and stents at the level of the lesion.

\section{Conflict of interest statement}

R. Ferraresi is a consultant for Abbott Vascular, Cook Medical, Medtronic, Terumo Medical Corporation, Cordis, and Biotronik. M. Manzi is a scientific board member for Abbott Vascular, and a proctor for Boston Scientific, Bard Medical, Cook Medical, and Medtronic. M. Roffi has received institutional research grants from Abbott Vascular, Biotronik, Biosensors, Boston Scientific, and Medtronic. R. Lorenzoni has no conflicts of interest to declare.

\section{References}

1. Kipling M, Mohammed A, Medding RN. Guidewires in clinical practice: applications and troubleshooting. Expert Rev Med Devices. 2009;6:187-95.

2. Ferraresi R, Palena LM, Mauri G, Manzi M. Tips and tricks for a correct “endo approach". J Cardiovasc Surg (Torino). 2013;54:685-711.

3. Bolia A, Miles KA, Brennan J, Bell PR. Percutaneous transluminal angioplasty of occlusions of the femoral and popliteal arteries by subintimal dissection. Cardiovasc Intervent Radiol. 1990;13:357-63.

4. Lorenzoni R, Lisi C, Lazzari M, Bovenzi F. Tools \& techniques: Above the knee angioplasty by transradial access. EuroIntervention. 2012;7:1118-9.

5. Schmidt A, Bausback Y, Piorkowski M, Werner M, Braunlich S, Ulrich M, Varcoe R, Friedenberger J, Schuster J, Botsios S, Scheinert D. Retrograde recanalization technique for use after failed antegrade angioplasty in chronic femoral artery occlusions. $J$ Endovasc Ther. 2012;19:23-9.

6. Manzi M, Fusaro M, Ceccacci T, Erente G, Dalla Paola L, Brocco E. Clinical results of below-the knee intervention using pedal-plantar loop technique for the revascularization of foot arteries. J Cardiovasc Surg (Torino). 2009;50:331-7.

7. Graziani L, Silvestro A, Monge L, Boffano GM, Kokaly F, Casadidio I, Giannini F. Transluminal angioplasty of peroneal artery branches in diabetics: initial technical experience. Cardiovasc Intervent Radiol. 2008;31:49-55.

8. Fusaro M, Tashani A, Mollichelli N, Medda M, Inglese L, BiondiZoccai GG. Retrograde pedal artery access for below-the-knee percutaneous revascularisation. J Cardiovasc Med (Hagerstown). 2007;8:216-8.

9. Palena LM, Manzi M. Extreme below-the-knee interventions: retrograde transmetatarsal or transplantar arch access for foot salvage in challenging cases of critical limb ischemia. J Endovasc Ther. 2012;19:805-11. 